Nouvelles perspectives en sciences sociales

Revue internationale de systémique complexe et d'études relationnelles

\title{
Le développement rural : une approche interdisciplinaire. Application à une région du sud-est tunisien
}

\section{Labiadh Ines}

Volume 7, numéro 1, octobre 2011

Sur le thème de l'interdisciplinarité

URI : https://id.erudit.org/iderudit/1007086ar

DOI : https://doi.org/10.7202/1007086ar

Aller au sommaire du numéro

Éditeur(s)

Prise de parole

ISSN

1712-8307 (imprimé)

1918-7475 (numérique)

Découvrir la revue

Citer cet article

Ines, L. (2011). Le développement rural : une approche interdisciplinaire. Application à une région du sud-est tunisien. Nouvelles perspectives en sciences sociales, 7(1), 157-182. https://doi.org/10.7202/1007086ar

\section{Résumé de l'article}

L'objectif de ce texte consiste à montrer qu'il existe des savoirs ou des disciplines proprement interdisciplinaires et ce en partant de l'affirmation de l'utilité de l'interdisciplinarité dans le traitement des problématiques complexes auxquels sont confrontés les chercheurs de nos jours. Dans le sud-est de la Tunisie, objet de l'étude, les résultats préliminaires de recherches permettent d'affirmer que le développement rural, en tant qu'approche de mise en valeur d'un territoire en difficulté, est un processus naturellement interdisciplinaire. 


\title{
Le développement rural : une approche interdisciplinaire. Application à une région du sud-est tunisien
}

\author{
LABIADH INES \\ Université de Grenoble
}

Les connaissances sont de plus en plus segmentées et les problèmes à résoudre de plus en plus complexes et globaux ${ }^{1}$.

'est ainsi que Edgar Morin justifie l'utilité de l'approche
interdisciplinaire pour le traitement des sujets et des pro-
blématiques complexes qui nécessitent un regard croisé provenant
de différentes disciplines. En effet, les disciplines provenant d'un
seul champ de savoir sont confrontées perpétuellement à des
contraintes dans la résolution de problèmes et l'apport de réponse
à des questionnements qui, même si situés à l'intérieur de leurs
frontières disciplinaires, nécessitent un regard extérieur pour être
résolus. Ce texte traite de la notion d'interdisciplinarité. Un
concept de plus en plus présent dans les différents débats autour
des méthodologies de travail en sciences sociales, naturelles ou
exactes. Qu'est-ce que l'interdisciplinarité? Quelles sont ses théo-
ries et qu'en est-il de ses champs d'application? L'objectif de ce

Edgar Morin, article du journal Le Monde de 27/02/1998 cité par Nadia Simony, Relais pour l'emploi : solidaires et acteurs de leur reclassement, Paris, Harmattan, 1998, p. 115. 
texte est de montrer qu'il existe des savoirs ou des disciplines proprement interdisciplinaires et ce, en partant de l'affirmation de l'utilité de l'interdisciplinarité dans le traitement des problématiques complexes auxquels sont confrontés les chercheurs de nos jours. Dans le sud-est de la Tunisie, objet de l'étude, les résultats préliminaires de nos recherches permettent d'affirmer que le développement rural, en tant qu'approche de mise en valeur d'un territoire en difficulté, est un processus naturellement interdisciplinaire.

\section{Interdisciplinarité : méthode de recherche}

La fécondité de l'interdisciplinarité comme méthode de recherche prend tout son sens dans la complexité des objets et sujets étudiés relevant de plusieurs disciplines. C'est ainsi que l'interdisciplinarité s'impose dès lors qu'on aborde un problème concret, un projet, un sujet ou un concept complexe.

Le concept de complexité renvoie à toutes les difficultés de compréhension (flou, incertain, imprévisible, ambigüe, aléatoire) que pose l'appréhension d'une réalité complexe et qui se traduisent pour l'observateur par un manque d'information ${ }^{2}$. En effet, certains problèmes échappent au domaine d'une seule discipline. Il s'agit souvent de problèmes complexes qui ne peuvent être étudiés et résolus que grâce à la coopération entre compétences propres à plusieurs disciplines ${ }^{3}$.

Par ailleurs, la prise de conscience de la complexité avait rompu avec la méthode cartésienne de réduction de la complexité héritée d'Aristote et rendue "opérationnelle " par Descartes au $17^{\mathrm{e}}$ siècle. «[...] Diviser chacune des difficultés que j’examinerais en autant de parcelles qu'il se pourrait et qu'il serait requis pour

2 Gérard Donnadieu, Daniel Durand, Danièle Neel, Emmanuel Nunez et Lionel Saint-Paul, L'approche systémique : de quoi s'agit-il, 2003, p. 3, [en ligne] http://www.afscet.asso.fr/SystemicApproach.pdf, consulté en décembre 2010

3 ICRA (Centre international pour la recherche agricole), équipe interdisciplinarité (2008), Interdisciplinarité, concepts-clés, [en ligne] http://www.icra-edu. org/objects/francolearn/ACFA0.pdf, en consulté en décembre 2010. 
les mieux résoudre " $"$. Cette méthode fragmente le savoir en autant de domaines qu'il y a de phénomènes à étudier.

Cette fragmentation du savoir entraîne une difficulté de communication grandissante entre les spécialistes et un isolement des disciplines scientifiques les unes envers les autres, d'une part, et face au monde réel, d'autre part. La méthode cartésienne de réduction de la complexité à ses composantes élémentaires n'est, en fait, adaptée qu'à l'étude de situations stables constituées d'un nombre limité d'éléments en interactions linéaires. C'est ainsi que de nombreuses études vont par la suite déceler les limites de cette méthode d'analyse tout en mettant l'accent sur la complexification, de plus en plus appréhendée, des éléments et des processus, qui relèvent de champs disciplinaires différents (économiques, culturels, politiques, technologiques, psychologiques, etc.).

Une autre approche est alors requise, fondée sur l'interaction entre différentes disciplines reliées par des relations d'influence et d'échange d'information. L'apport de l'interdisciplinarité, dans ce sens, provient de sa capacité à associer autour d'un même objet d'étude différentes disciplines sans pour autant faire perdre à chacune ses normes, théories et objectifs spécifiques. Mais pourquoi rassembler différentes disciplines autour d'un objet central que chacune pourrait étudier indépendamment?

C'est en fait l'invisibilité de la solution pour un problème au sein d'une discipline qui fait appel par la suite à un affranchissement des frontières de cette dernière et au dépassement des limites de son savoir. L'encastrement de ce savoir entre des normes et des concepts unidisciplinaires le rend, en fait, limité et incapable par la suite de servir pour voir les choses au-delà de ses frontières. C'est ainsi que la résolution des problèmes qui chevauchent les disciplines nécessite forcément une intégration des analyses et des travaux émanant des différentes disciplines concernées. Dès lors, les spécificités de chaque discipline (son objet d'étude, le type de ces questions et l'interprétation de ces

$4 \quad$ René Descartes, Discours de la méthode, Paris, Hachette, 1856, 53 p., [en ligne] http://books.google.fr, consulté en janvier 2011. 
résultats) se dissolvent dans un objectif central et une perspective convergente.

L'esprit de travail passe de l'hyperdisciplinarité ou de l'« hyperspécialisation " ${ }^{5}$ de chacun à la pluridisciplinarité et à la tolérance d'autres visions et réflexions, certes différentes, mais convergeant vers un même objet central. L'interdisciplinarité serait alors une connaissance capable de saisir ce qui est tissé ensemble ${ }^{6}$. C'est en ce sens qu'un proverbe malien explique qu'on ne peut pas ramasser une pierre avec un seul doigt. Il faut pour ceci recourir aux cinq doigts et donc à toute la main. De même pour aboutir à la résolution d'un problème, à la compréhension d'un phénomène ou à la mise en place d'idées nouvelles, il faut développer une capacité de synthèse et d'analyse à partir de la collaboration et de l'accord entre les perspectives de plusieurs disciplines rassemblées autour d'un objet central d'étude. La vision de ce dernier devient dès lors plus globale et généralisée en identifiant et en intégrant toutes les relations entre les différents éléments impliqués.

L'interdisciplinarité comme méthode de recherche s'oppose dans ses principes au travail individuel et au confinement de la discipline sur elle-même; elle prône par ailleurs le travail collectif, l'échange et l'ouverture d'esprit en vue de mieux cerner l'objet d'étude dans sa complexité et ses divers éléments constitutifs. Il faut toutefois s'assurer avant de recourir à une approche interdisciplinaire que cet objet ou ce phénomène est partagé et présente un intérêt majeur pour chacune des disciplines impliquées; en fait, on ne peut pas faire appel à une discipline ou même à un simple concept dans une démarche de travail interdisciplinaire sans s'assurer de la qualité de son apport, que ce dernier touche de près ou de loin l'objet à l'étude. C'est dans ce registre qu'un médecin examinant un malade qui souffre d'un mal de tête étrange peut recourir à la psychologie pour comprendre la cause

5 Terme emprunté à Edgar Morin, Sur l'interdisciplinarité, 2003, p. 5, [en ligne] http://www.mcxapc.org/docs/conseilscient/morin_interdisciplinarite_021103.pdf, consulté len novembre 2010.

6 Edgar Morin et Jean-Louis, Le Moigne, L'intelligence de la complexité, Paris, Harmattan, 1999, 332 p. 
de cette souffrance anatomique. De même, le climatologue fait appel aux lois de la physique pour expliquer l'accentuation du phénomène des gaz à effet de serre.

Retenons ici qu'une seule discipline ne peut tout expliquer, surtout lorsqu'on aborde des questions complexes. La complexité appelle des stratégies interdisciplinaires. La discipline, dans sa quête continue de réponses et d'explications, doit dépasser ses frontières pour essayer de trouver une réponse chez d'autres disciplines. Cette circulation de notions et concepts que Edgar Morin nommait "migration " est la seule garante de la résolution des problèmes qui échappent aux disciplines et qui, par la suite, nécessitent une collaboration avec d'autres champs de savoir concernés eux-mêmes par ces problèmes.

\section{Interdisciplinarité : une ingénierie systémique}

L'interdisciplinarité, comme on vient de la définir, implique une association de compétences utilisées en vue d'une réalisation commune d'un travail, d'une étude ou d'un projet. Théoriquement, l'interdisciplinarité doit opter pour l'approche systémique dans sa démarche. On essayera, dans ce qui suit, de justifier cette idée.

Par définition, "l'approche systémique s'appuie sur une approche globale des problèmes ou des systèmes que l'on étudie et se concentre sur le jeu des interactions entre les éléments ${ }^{8} »$. Il s'agit d'un champ interdisciplinaire servant à l'étude des objets complexes ou multidimensionnels et dont l'étude par une approche linéaire ou " habituelle " ne permet pas de traiter la question en sa totalité .

L'approche linéaire consiste à trouver une solution simple et immédiate à un problème donné en supposant qu’à ce problème il existe une cause antécédente précise. On tombe ainsi le plus

\footnotetext{
Edgar Morin, op.cit., p. 6.

8 Joël De Rosnay, Le macroscope (Vers une vision globale), Paris, Seuil, 1975, $295 \mathrm{p}$.

9 Wikipédia, L'encyclopédie libre en ligne (2011), Approche systémique, [en ligne] http://fr.wikipedia.org/wiki/Approche_systémique, consulté en janvier 2011.
} 
souvent dans le piège de la "banalisation " des problèmes en oubliant que certains objets, situations ou sujets d'étude, de par leur complexité, nécessitent une approche systémique.

Cette dernière traite le sujet dans un contexte général et permet de déduire les différents éléments du système dans lequel il s'inscrit. L'approche systémique part donc du problème observé, de la constatation faite ou de la question posée pour arriver enfin à différentes hypothèses explicatives pouvant être à l'origine du problème à résoudre. Il s'agit d'une démarche globale qui traite de sujets complexes faisant partie d'un système dans lequel ils interagissent avec d'autres éléments dans des liens de causes à effets. C'est le cas, par exemple, de la contribution de l'anthropologie à la compréhension d'un phénomène humain donné qu'elle essaye de rapporter au contexte défini dans lequel il a pris naissance. C'est ainsi que la forte immigration observée au niveau d'une région donnée peut être expliquée par le taux de chômage important dans cette région, la pauvreté élevée, la crise d'activités à l'origine rentables, la facilité de franchissement des frontières, et par d'autres phénomènes explicatifs faisant appel à des champs de savoir différents.

La méthode interdisciplinaire de recherche est indispensable du moment où les sujets qu'elle traite sont complexes et parfois même inextricables à tel point qu'il devient difficile de distinguer leurs différents éléments constitutifs ainsi que les facteurs ou constituants du même système avec lesquels ils interagissent. C'est dans ce registre que l'interdisciplinarité pratique naturellement la systémique. Elle réunit différentes disciplines autour d'un objet central d'étude; le tout forme un système de savoirs et de visions apte à expliquer, à traduire et à comprendre cet objet. C'est en fait le principe de l'interaction ${ }^{10}$ caractérisant l'approche systémique qui permet la formation de ce système où chaque discipline peut s'informer et agir sur l'état des autres disciplines. L'interaction entre les éléments de ce système -et donc les différentes disciplines dans notre cas- se traduit souvent

$10 \quad$ Michel Crozier et Erhard Friedberg, L'acteur et le système, les contraintes de l'action collective, Paris, Seuil, 1992, 500 p. 
par un rapport d'influence ou d'échange portant sur des flux d'informations, de valeurs, de principes, de théories et même de méthodologies.

Les chercheurs et les praticiens sont appelés lors d'un travail interdisciplinaire à respecter les démarches d'une approche globale. Cette dernière prône le travail collectif au sein d'un système de spécialistes provenant de différentes disciplines. Mais comment parvenir à réussir cette approche systémique dans une équipe formée de membres aussi différents que les champs disciplinaires qu'ils maitrisent? Le secret réside dans la connaissance des objets et concepts des autres disciplines, dans l'attachement à l'échange entre les différentes disciplines plutôt qu'à l'analyse à la loupe de l'apport unidisciplinaire et dans le raisonnement par rapport à l'objectif du système.

L'approche systémique fait, certes, large part à des connaissances théoriques, mais aussi au savoir-faire, à l'apprentissage et au partenariat. C'est ainsi que la mise en ouvre de cette démarche prône l'effort d'apprentissage conceptuel et pratique que doit fournir les personnes impliquées dans l'appréhension d'une réalité multidimensionnelle. La complexification des éléments et des processus, qui relèvent de champs différents (économiques, culturels, politiques, technologiques, psychologiques, etc.) pourra par la suite être surmontée pour atteindre la finalité, l'objectif ou le but annoncé par le système d'étude. Cette finalité constitue, par ailleurs, un des concepts de base de l'approche systémique. En effet, en procédant par une approche systémique, l'homme, (chercheur, décideur, politicien, citoyen, etc.) se penche sur une réalité complexe qu'il essaye par la suite de comprendre et d'assimiler avec des connaissances, des pensées et des visions elles aussi systémiques. Cette manière d'aborder un sujet aboutit à un objectif déjà fixé au préalable. Il s'agit de comprendre un phénomène complexe, de s'y orienter dans un premier temps et, dans un second temps, d'agir sur lui.

Il faut ainsi revenir perpétuellement à l'objet central du travail interdisciplinaire sans pour autant oublier qu'il ne peut être atteint sans la contribution des autres disciplines et donc des 
autres éléments du système. Rappelons dans ce registre avec Jean-Claude Lugan qu'à partir d'un certain seuil, l'approfondissement des connaissances sur un secteur étroit de la réalité perd de son efficacité si ce secteur n'est pas réintroduit dans des ensembles plus vastes ${ }^{11}$.

Autrement dit, si le travail individuel disciplinaire est important pour la création des savoirs, le travail en collaboration avec d'autres disciplines permet une meilleure compréhension des problèmes. Par ailleurs, le système formé de différentes disciplines autour d'un objet central permet à chacune d'entre elles de se comprendre mieux et de comprendre aussi les autres sciences. Certes, en prenant de la distance avec ses outils et ses méthodes et les comparant avec d'autres, le chercheur arrive à comprendre mieux sa propre discipline. Il faut rappeler toutefois que pour être compétente, une équipe interdisciplinaire doit être formée de membres compétents chacun dans sa discipline.

Ce recours en même temps à des disciplines, parfois très diverses, qu'exigent certains processus de complexification de champs de recherche disciplinaire peut être justifié par la nécessité de se référer à des savoirs différents pour pouvoir traiter le sujet d'étude dans tous ses angles. Cette vision systémique de l'approche interdisciplinaire peut être schématisée à la manière de la figure 1.

Toute idée, concept ou réflexion schématisé dans la figure 1 fait appel à une discipline à part. Les interactions entre les différents éléments du système nécessitent forcément un chevauchement entre les différentes disciplines à l'origine de ces éléments. C'est ainsi qu'on se retrouve avec un travail où se combinent les théories, les objets de recherche et les méthodologies de différentes disciplines concernées. Ceci crée une richesse interdisciplinaire dont le degré peut varier avec le nombre des disciplines impliquées, la "distance " qui les sépare, la nouveauté et la créativité créées par la combinaison de leurs éléments disciplinaires et le degré d'intégration entre elles ${ }^{12}$.

11 Jean Claude Lugan, La systémique sociale, Paris, PUF, 2005, 127 p.

12 Moti Nissani, "Fruits, Salads, and Smoothies: A Working Definition of 
Figure 1

Interaction entre les différents éléments d'un système

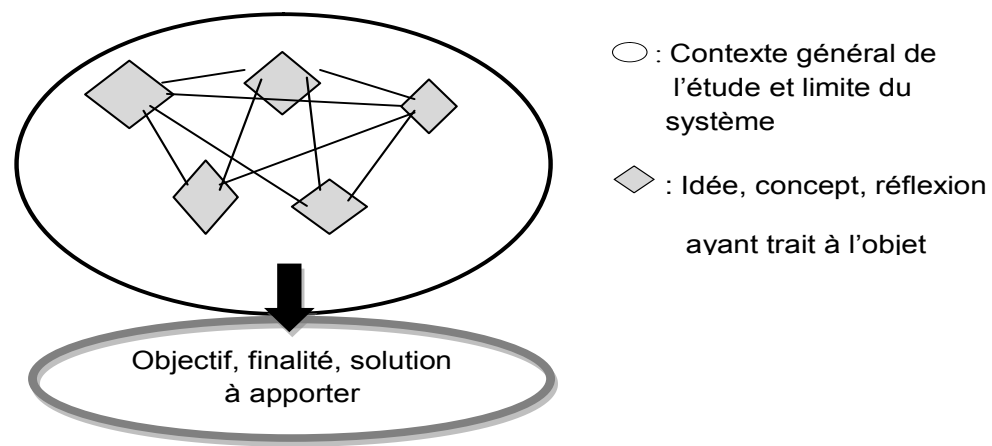

L'exemple de l'étude du phénomène migratoire au niveau d'une région donnée peut être repris dans ce cadre. Ainsi, les différentes explications pouvant être données à ce phénomène se rapportent chacune à une discipline bien déterminée. Si le chômage est une affaire sociologique, le taux de pauvreté est étudié par les démographes. Pour sa part, la crise des activités à l'origine rentables dans la région est un facteur principalement économique. Par ailleurs, la facilité de franchissement des frontières peut être étudiée d'un point de vue géographique ou même politique. La compréhension de la raison pour laquelle une telle région est qualifiée de région "à forte immigration " passe par la prise en considération de l'apport de chacun des savoirs déjà cités dans cette question, d'une part. D'autre part, la réponse doit être globale et conforme à une étude implantée dans un système de données où tous les éléments sont en interaction continue.

Ces interactions multiples et variées entre disciplines expriment le principe de totalité ou de globalité caractérisant l'approche systémique. Un objet complexe à étudier ne peut pas être, en effet, réduit à la somme des éléments qui le constituent ${ }^{13}$, chaque

Interdisciplinarity ", Journal of Educational Thought, vol. 29, 1995, p 119126.

13 Ludwig Von Bertalanffy, Théorie générale des systèmes, Paris, Dunod/ Systémique, 1993, 308 p. 
discipline ne pouvant pas à elle seule expliquer le phénomène observé ou rapporter une solution à la problématique posée, son domaine d'étude et de compétences étant limité. Il convient, donc, d'aborder tous les aspects d'un problème progressivement, mais non séquentiellement ${ }^{14}$, ce qui revient à partir d'une vue générale (globale) pour approfondir ensuite les détails.

Ce principe de globalité appelle à examiner les propriétés de tout le système qu'on ne peut pas réduire aux propriétés de ses parties. Il est basé sur la théorie holiste qui traite le système complexe comme étant une entité possédant des caractéristiques liées à sa totalité, et des propriétés non déductibles de celles de ses éléments. Cette vision liée à l'approche systémique s'oppose à la théorie individualiste dans laquelle la compréhension du " tout » doit passer forcément par l'étude de ses parties. Retenons ici que l'interdisciplinarité est théoriquement une ingénierie systémique dont la finalité consiste à comprendre un sujet donné qui doit être envisagé en sa totalité et par le biais de différentes disciplines et champs de compétences en interaction continue.

\section{Approche interdisciplinaire au développement rural : application à la région de Béni Khédache au sud-est tunisien}

3.1. Entre problématisation et conception des actions de développement rural : quelle place pour l'interdisciplinarité?

Le développement rural est une approche globale et cordonnée des territoires ruraux dans leurs diverses composantes. Ainsi il prend en considération les différents aspects liés à la société et au milieu dans lequel il intervient. Pour ce faire, sont intégrés le volet social (démographie, traditions, coutumes, services, etc.), économique (activités productives, emploi, ressources valorisables, etc.) et environnemental (climat, sol, végétation, etc.) eafin de créer une nouvelle image du rural (image souhaitée du futur) en partant d'une image de référence (réalité présente du milieu).

Une bonne étude du milieu rural exige de bons spécialistes

14 Gérard Donnadieu et al., op. cit., p. 4. 
capables d'ouverture. En effet, le rural peut être considéré comme un système " objet complexe, organisé, formé d'éléments, d'individus et d'actions en interaction dynamique ${ }^{15}$. Dans ce sens, il est le lieu physique d'application de plusieurs disciplines (sociologie, agronomie, écologie, géographie, etc.) et il constitue un sujet de rencontre et de collaboration entre les différents chercheurs et praticiens, chose qui fait de lui un vrai dossier d'interdisciplinarité. L'idée est donc de créer une formation complémentaire qui apporte aux spécialistes une culture générale interdisciplinaire et une large ouverture d'esprit sur le thème du développement rural. L'écologiste, par exemple, doit comprendre un problème économique et l'économiste doit savoir ce qu'est la biosphère. De même, le sociologue doit avoir une idée à propos des saisons de cultures et l'agronome à propos des phénomènes de chômage et d'analphabétisme.

Le développement rural apparaît donc comme un savoir interdisciplinaire où les diagnostics et les plans d'actions proposés pour un milieu donné constituent la résultante d'un travail collectif et pluridisciplinaire. Ainsi, la mise en place d'un projet de développement rural débute par un diagnostic du territoire qui servira comme base de jugements et de réflexions aboutissant à des actions et à des projets de développement en faveur de ce milieu rural. La concrétisation d'un projet de développement rural relève d'une vision globale du milieu et nécessite la prise en compte de la caractéristique multidimensionnelle du rural dans la conception des actions et des interventions à mener.

3.2. Application de l'interdisciplinarité du développement rural à une région du sud-est tunisien

\subsubsection{Développement rural en Tunisie}

Le développement rural en Tunisie a fait pour longtemps l'objet de différents programmes mis en place par le planificateur. Ces programmes étaient destinés en premier lieu aux régions les plus

15 Définition synthétique de celles données par Michel Crozier, Jean Ladrière et Edgar Morin dans Daniel Durand, La Systémique, Paris, PUF, [1979] 1998, $121 \mathrm{p}$. 
nécessiteuses, dites "zones difficiles » ou encore "zones défavorisées ". Il s'agit des zones auxquelles on peut assigner le terme " retard de développement » et qui, tout en étant intégrées dans des systèmes d'interdépendance avec les zones développées, subissent le plus souvent des formes de marginalité ${ }^{16}$.

À travers ces programmes, le planificateur vise à atteindre les objectifs arrêtés par les plans quinquennaux de développement parmi lesquels nous trouvons essentiellement l'équilibre régional. Ils visent directement les conditions de vie de la population et se sont inspirés d'une logique économique de rentabilité des investissements. Ainsi, la Tunisie a opté pour différents programmes dont le Programme de développement rural intégré (PDRI), le Programme de développement agricole intégré (PDAI), le Programme de céveloppement urbain intégré (PDUI), le Fond de solidarité nationale (FSN), le Programme régional pour le développement (PRD), etc.

Ces différents programmes ou projets de développement avaient comme objectif principal la lutte contre la pauvreté dans les zones les moins développées du pays et ce, en optant pour des politiques d'amélioration des activités agricoles, de gestion des ressources naturelles et de création des activités génératrices de revenus pour les populations pauvres souffrant du chômage et d'exclusion.

Les schémas dessinés pour ces espaces étaient au début tous pareils dans le sens où les «zones difficiles » étaient considérées comme une entité homogène où les problèmes similaires étaient résolus par des approches générales et pareilles en allant d'un territoire défavorisé à un autre. En effet, l'importance de l'agriculture dans l'économie du monde rural a fait que les premières politiques de développement en faveur de l'espace rural ont été essentiellement basées sur le développement du secteur agricole qui doit générer des revenus suffisants pour permettre aux ruraux des conditions de vie décentes. Par la suite, l'échec de cette

16 Mongi Sghaier et Ali Abbab, "L'inscription de la thématique du colloque DELZOD dans le parcours scientifique du laboratoire d'Économie et sociétés rurales ", Cadrage du colloque international "Sociétés en transition et développement local en Zones difficiles, DELZOD ", Médenine, IRA, 2009. 
politique et surtout l'aggravation des déséquilibres entre le milieu rural et urbain ont conduit à accorder une plus grande importance au développement rural au sens large du terme avec à la fois des actions de développement de l'infrastructure de développement de l'agriculture et parallèlement de toute activité porteuse d'espoir de développement socio-économique ${ }^{17}$.

Une seconde génération de politiques de développement rural est par la suite apparu en Tunisie; des politiques de plus en plus destinées à des secteurs économiques autres que l'agriculture et qui réussissaient justement à mettre en place une économie diversifiée dans les "zones difficiles ». Le développement rural devient par la suite plurisectoriel et de plus en plus autonome par rapport au développement agricole.

La pluri-sectorialité du développement rural implique une intégration de toutes les richesses dont est doté le territoire. Dans ce sens, l'articulation entre les différents secteurs d'activité devient indispensable pour une meilleure allocation $\mathrm{ds}$ ressources locales. Les activités portées par un projet de développement doivent toucher aux différentes dimensions de la société à savoir le social, l'économique et le culturel. Par ailleurs, force est de signaler, que dans les milieux ruraux où l'agriculture constitue dans la plupart du temps l'activité économique principale, il est presque impossible de remédier à la pression sur les ressources naturelles exercée par la population. En effet, l'absence d'alternatives à cette activité -en lien avec la faible diversification du tissu économique- fait que les populations se tournent toujours vers le travail de la terre comme source principale de revenu et qu'elles continuent par la suite à puiser dans les ressources naturelles (eau, sol, végétation) d'une manière le plus souvent irraisonnable et avec des pratiques agricoles et pastorales généralement inadéquates ${ }^{18}$. Le développement rural doit cibler aussi

\footnotetext{
17 Mohamed Elloumi, «Politiques du développement rural en Tunisie, acquis et perspectives ", Options Méditerranéennes, Sér. A / n 71, 2006, p. 55-65.

18 Mongi Sghaier, Ali Abaab et Pierre Campagne, "L'expérimentation de nouvelles approches de développement local et de gestion participative des ressources naturelles ", dans Didier Genin, Henri Guillaume, Mohammed Ouessar, Azalez Ouled Belgacem, Bruno Romagny, Mongi Sghaier et
} 
la protection des ressources naturelles et la mise en ouvre de meilleurs schémas pour une gestion durable de ces dernières.

Dans un autre registre, le développement des territoires ruraux est un processus polydimensionnel où les dimensions des autres sciences se trouvent présentes et où la perspective globale, loin d'être chassée par la multiplicité des perspectives particulières, est requise par celle-ci. C'est un processus non seulement économique, mais aussi sociologique, écologique et culturel. En fait, la constitution d'un projet interdisciplinaire permet de créer l'échange, la coopération et la polycompétence issue de l'assemblée autour d'une même table de différentes disciplines en lien avec le développement d'un territoire en difficulté, les notionsclés impliquées étant coopération articulation, objet commun et projet commun ${ }^{19}$.

\subsubsection{Carte d'identité du territoire d'étude}

La délégation de Béni Khédache est créée administrativement en 1956, date de l'indépendance du pays. Située au nord-ouest du gouvernorat de Médenine, lequel se trouve dans le sud-est du pays (figure 2), Béni Khédache couvre une superficie de 1356 $\mathrm{km}^{2}$ et comptait 28600 habitants en 2004. Son climat est semiaride et marqué par la variabilité. En effet, cette région est soumise à la fois aux influences provenant de la zone côtière ainsi qu'à celles du désert. À cela s'ajoutent les influences du relief sur les différents paramètres climatiques, notamment la température, la pluie et le vent. La région est soumise à deux régimes de vents dominants; les vents de secteur nord, nord-est à est, en provenance de la côte et les vents de secteur sud, sud-ouest à ouest, en provenance du désert ${ }^{20}$.

Houcline Taamallah (dir.), Entre désertification et développement. La Jeffara tunisienne, Tunis, Éditions Cérès, IRD et IRA, 2006, p. 303-313.

20 Institut des Régions Arides (IRA) et Coopération Technique Allemande (GTZ), Plan d'action local de lutte contre la désertification (PALLCD) dans la Délégation de Béni Khédache (Gouvernorat de Médenine), Ministère de l'environnement et du développement durable, 2007, 135 p. 
La variabilité du climat se manifeste à travers le régime pluviométrique caractérisé par des précipitations faibles et irrégulières, parfois torrentielles. La pluviométrie moyenne est de 230 $\mathrm{mm} / \mathrm{an}$, mais elle peut aller de 90 jusqu'à $700 \mathrm{~mm} / \mathrm{an}$. Cette moyenne annuelle ne reflète pas la grande irrégularité interannuelle des pluies. Quant au régime thermique, il est caractérisé par des températures élevées (en moyenne $38^{\circ} \mathrm{C}$ ) durant l'été, et des températures relativement fraîches durant l'hiver (en moyenne $\left.18^{\circ} \mathrm{C}\right)$.

Figure $2^{21}$

\section{Béni Khédache au sud-est de la Tunisie}

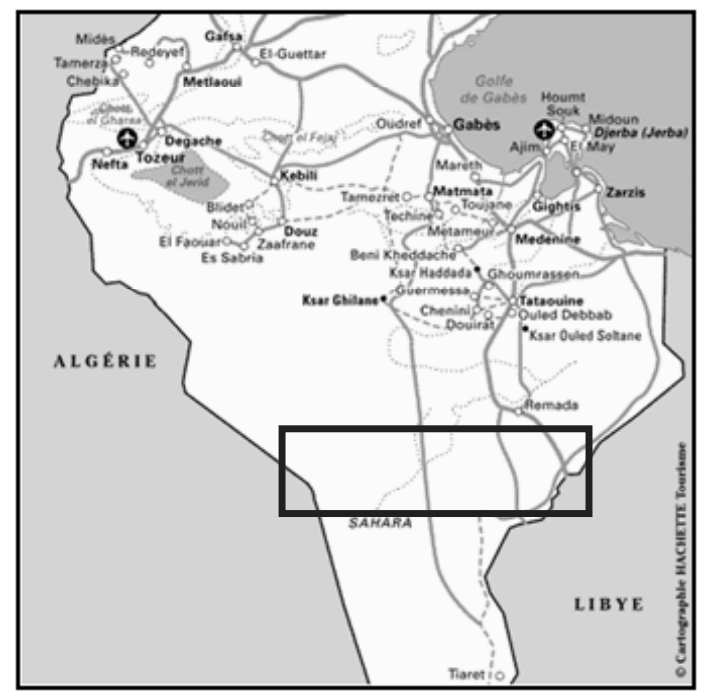

La délégation de Béni Khédache, région de notre étude, est une zone montagneuse aride qui constitue une des régions défavorisées de la Tunisie et qui confrontée à plusieurs difficultés pour suivre un processus de développement. Ces difficultés sont liées essentiellement aux contraintes climatiques et à la rareté des ressources naturelles.

21 Carte de la Tunisie (2010), adaptée, [en ligne] http://www.routard.com/ guide_carte/code_dest/tunisie.htm, consulté en décembre 2010. 
L'économie de la région est basée principalement sur l'agriculture. Cette activité demeure l'activité qui occupe et qui occupera, sans doute pendant longtemps encore, la majeure partie des populations de cette région comme cela se produit dans les régions marginalisées des pays en voie de développement, et ce, malgré les contraintes climatiques du milieu.

L'économie locale de la région, basée principalement sur l'agriculture, connaît une faiblesse de son tissu et des activités à revenus insuffisants pour satisfaire aux nouveaux besoins de développement d'une population en croissance continue. La faiblesse du tissu économique est due essentiellement à l'enclavement géographique de la zone d'où les difficultés de commercialisation et d'écoulement des produits, d'une part. D'autre part, les activités économiques sont peu diversifiées, certains ménages essayent de diversifier leurs sources de revenu par de petits métiers de commerce ou d'artisanat mais les revenus restent toujours minimes. Ceci est dû essentiellement à l'insuffisance de la demande locale, au niveau faible de l'organisation de la filière et au manque d'esprit de marketing. En effet, la population de Béni Khédache est caractérisée par l'absence d'esprit commercial dans l'unité de production familiale en raison d'une culture de gestion de risque basée sur l'autoconsommation, la conservation et la réserve des produits ${ }^{22}$.

L'économie peu diversifiée de la région et l'activité agricole contrariée par un climat aride et des ressources en eau de plus en plus rares ont conduit à deux phénomènes sociaux majeurs : l'immigration et le chômage. Le chômage touche de plus en plus les jeunes diplômés, y compris les filles, qui se déplacent par conséquent vers les villes côtières pour travailler dans les usines de textile. La région de Béni Khédache est une région de forte émigration. La population locale, notamment du sexe masculin, quitte le territoire à la recherche d'horizons de travail plus ouverts ailleurs. Cette tendance vers l'émigration s'est traduite par un

22 Hanen Abichou, Mongi Sghaier et Anne-marie Jouve, "Quelle alternative pour le développement des zones défavorisées en Tunisie », New Medit, vol. 7, $\mathrm{n}^{\circ} 1$, mars 2008, p. 38-44. 
taux de croissance démographique négatif et une prédominance du sexe féminin.

\subsubsection{Problématisation de la région}

Conscients de la situation difficile dans laquelle vit la population de Béni Khédache, les acteurs locaux (chercheurs, développeurs, aménageurs, etc.) se sont mobilisés avec des aides et des appuis extérieurs afin d'améliorer les conditions de vie des ruraux et ce, en s'appuyant sur une logique économique d'accroissement des investissements et des richesses.

Ce travail, qui n'est autre qu'un processus de développement rural, avait commencé par un diagnostic biophysique et socioéconomique de la région ayant permis de constituer la problématisation de la région pour ensuite se baser sur les résultats de ce diagnostic dans la proposition des actions et des solutions aux problèmes posés.

La concrétisation du diagnostic de la région par une équipe de chercheurs et de spécialistes interdisciplinaires a duré presque cinq mois. Dans ce cadre, les spécialistes (sociologues, économistes, aménageurs, planificateurs, agronomes, etc.) avaient opéré en parfaite concertation et intégration de rapports.

La problématisation de la région de Béni Khédache (tableau 1) avait été élaborée à la base de connaissances formelles issues des chercheurs et des connaissances pratiques apportées par des hommes de terrain.

Le tableau 1 résume les résultats ${ }^{23}$ de travaux de recherche et de terrain menés par une équipe multidisciplinaire composée de personnes rattachées à différentes institutions, laboratoires de recherche, directions et services étatiques implantés dans le gouvernorat de Médenine et dans la délégation de Béni Khédache.

$\overline{23}$ L'étude menée est beaucoup plus exhaustive que le tableau rapporté ici. Notre ambition étant de montrer que le travail avait été réalisé avec la contribution de différentes disciplines; nous nous sommes contentés, pour ce faire, du minimum des résultats dégagés. 


\section{Tableau 1}

Problématisation de la région de Béni Khédache

\begin{tabular}{|c|c|c|}
\hline Le secteur agricole & Le secteur économique & La situation sociale \\
\hline $\begin{array}{l}\text { Mobilisation et } \\
\text { valorisation des } \\
\text { ressources naturelles } \\
\text { ayant atteint un } \\
\text { niveau critique, ce } \\
\text { qui limite le } \\
\text { développement } \\
\text { agricole et pastoral } \\
\text { Dégradation } \\
\text { continue qui touche } \\
\text { à la fois le sol, les } \\
\text { ressources végétales } \\
\text { et les ressources en } \\
\text { eau } \\
\text { Productivité agricole } \\
\text { faible et qualité des } \\
\text { produits agricoles de } \\
\text { plus en plus } \\
\text { médiocre }\end{array}$ & $\begin{aligned} & \begin{array}{l}\text { Enclavement } \\
\text { géographique et }\end{array} \\
& \text { économique } \\
&>\text { Difficultés } \\
& \text { d'écoulement et de } \\
& \text { commercialisation } \\
& \text { des produits } \\
&>\text { Absence de } \\
& \text { l'organisation de la } \\
& \text { filière } \\
&>\text { Manque de l'esprit } \\
& \text { de marketing* }\end{aligned}$ & $\begin{array}{l}>\text { Pourcentage élevé de } \\
\text { familles nécessiteuses } \\
\text { Importance des } \\
\text { phénomènes } \\
\text { migratoire et d'exode } \\
>\text { Taux de chômage } \\
\text { élevé et } \\
\text { augmentation des } \\
\text { demandes d'emploi }\end{array}$ \\
\hline
\end{tabular}

\section{* Production destinée principalement à la consommation familiale}

Le diagnostic élaboré sur la région de Béni Khédache touchait deux volets. Le premier traite de l'aspect biophysique de la région et le deuxième la constituante socio-économique. L'élaboration de ce diagnostic s'est opérée par deux moyens :

1) La collecte d'informations et la recherche bibliographique auprès des principales personnes sources, notamment les membres du personnel du CRDA ${ }^{24}$, des services locaux de l'agriculture, de l'ODS ${ }^{25}$, de l'IRA, etc.

2) Des investigations de terrain, en concertation avec les acteurs locaux et la collecte de données complémentaires auprès des principaux partenaires, notamment le CRDA de Médenine, les autorités locales et les services techniques dans la délégation de Béni Khédache. Ceci était principalement l'affaire des hommes de terrain qui, à travers le contact direct avec la population et les différents acteurs locaux dans la région, avaient réussi à se pro-

\footnotetext{
24 Commissariat régional du développement agricole

25 Office de développement du sud
} 
curer les données qui ne pouvaient pas être acquises par une simple recherche bibliographique.

Dans l'élaboration de ce diagnostic pour la région, des chercheurs provenant des institutions de recherche, d'une part, et, d'autre part, des ingénieurs et hommes de terrain travaillant dans les services techniques à Béni Khédache ont collaboré pour donner une image commune reflétant la réalité du terrain à l'étude, objet central de leurs travaux.

En ce sens, les agronomes, les membres du personnel du CRDA et les agriculteurs de la région se sont intéressés à la problématique du secteur agricole à Béni Khédache. Chacun avait contribué à ce sujet de par son savoir disciplinaire ou son expérience et ses connaissances du terrain. Le résultat consiste en une carte de connaissances sur l'évolution du secteur agricole à Béni Khédache et notamment sur la crise que connaissent l'agriculture et l'élevage ces dernières années. Des discussions, des enquêtes sur le terrain avec des agriculteurs et des réunions au siège du CRDA ont permis de constituer la base des données concernant le secteur agricole dans la région et qui servait comme outil d'aide à la décision dans l'élaboration du programme de développement agricole à Béni Khédache.

Pour leur part, les économistes se sont penchés sur l'étude du secteur économique dans la région. Ainsi ont été menées des études sur les principaux secteurs productifs (agriculture, industrie, secteur artisanal, commerce, tourisme) en vue de déceler les défaillances de l'économie de la région et de comprendre le fonctionnement actuel des systèmes productifs.

Pour ce qui est de l'aspect social, les sociologues sont partis de deux observations que n'importe quel œil, même extérieur à la région, peut extraire en visitant Béni Khédache. Il s'agit du phénomène migratoire frappant et du taux de chômage important. Leurs travaux se sont basés sur des données statistiques qui traitent d'immigration et d'émigration au niveau du territoire d'étude, et des demandes d'emploi enregistrées dans la délégation. 
Les agronomes, les économistes et sociologues ne sont pas basés uniquement sur des données statistiques pour formuler leurs rapports, mais aussi sur des observations et des prospections faites directement sur le terrain auprès de la population locale et des services régionaux à Béni Khédache.

Il est à signaler que les membres de l'équipe de travail ont été divisés au préalable pour travailler sur cinq grandes thématiques : agriculture, $\mathrm{CES}^{26}$, parcours et élevage, activités non agricoles, et institutions et infrastructure. Chaque équipe s'est alors penchée sur un aspect particulier en lien avec le territoire d'étude pour aboutir à l'identification des problèmes à résoudre, des objectifs à atteindre, des activités à entreprendre, des impacts attendus et ce, par thématique de travail.

Une fois les travaux de chaque équipe terminés, les rapports des trois groupes de travail ont été rassemblés en un seul qui représente un diagnostic de la situation générale au niveau de la région de Béni Khédache. Le rassemblement des différents rapports thématiques en un seul s'est fait d'une manière intégrée suite à l'organisation de réunions de concertation avec les principaux partenaires et acteurs locaux concernés par le développement de Béni Khédache.

Au cours de ces réunions, les résultats des diagnostics, biophysique et socio-économique, de la zone ont été exposés à ces partenaires et ont fait l'objet de recommandations, d'orientations et de suggestions ayant aboutit au rapport final. Ce dernier, nommé "Plan d'action local de lutte contre la désertification (PALLCD) dans la délégation de Béni Khédache ", est actuellement considéré comme un cadre stratégique pour l'initiation d'un processus de développement durable de la délégation.

Cette réalisation commune des résultats constitue une réelle assise interdisciplinaire qui rassemble à la fois agronomes, sociologues et économistes autour d'un objet central d'étude : les causes des conditions difficiles dans lesquelles vit la population de la région. C'est ainsi que ce travail collectif a permis un chevauchement entre les différentes disciplines impliquées et la

26 CES : Conservation des eaux et des sols 
compréhension de phénomènes ou de réalités parfois assez pluridimensionnelles.

À titre d'exemple, le groupe travaillant sur la thématique agriculture, avait conclu que l'activité agricole à Béni Khédache connaissait une réelle crise qui se manifestait par un abandon progressif des terres agricoles et par une tendance accrue à l'immigration enregistrée notamment auprès des jeunes. Ces derniers manifestent en fait, un désintéressement important pour les activités de la terre, chose qui a été expliquée par un certain nombre d'hypothèses :

- Les jeunes à Béni Khédache sont de plus en plus instruits et les études qu'ils font doivent déboucher sur un travail selon eux, "plus prestigieux ", mais surtout plus lié aux spécialités de leurs études le plus souvent éloignées de l'agriculture;

- La faible rentabilité des activités agricoles ces dernières années les démotive à se tourner vers le travail de la terre;

- La possibilité qui leur est offerte de quitter le pays, que ce soit d'une manière légale ou clandestine, les séduit.

C'est ainsi que la réalité de l'agriculture qu'avait affiché le groupe travaillant sur cette thématique avait été le point de départ pour des réflexions et des hypothèses émanant des autres groupes thématiques et des différents partenaires provenant de disciplines variées. Dans ce cadre, la sociologie était incapable à elle seule d'expliquer les taux de plus en plus élevés d'immigration des jeunes malgré les restrictions appliquées par les pays de destination. Il a fallu, pour ce faire, comprendre que l'activité principale dans la région (l'agriculture) était en crise. Cette idée de crise de l'agriculture, définie à la fois par des agronomes et des économistes, est venue appuyer l'observation faite par des sociologues sur les jeunes de la région de plus en plus instruits et conscients que leurs ambitions en termes de travail sont de moins en moins implantées dans leur territoire d'origine.

C'est en fait le décloisonnement de ces différentes disciplines qui a permis la reconstruction de la réalité morcelée sur Béni Khédache, et ce, par la composition d'un groupe multidiscipli- 
naire pour travailler sur un même thème, chacun traitant un aspect particulier ${ }^{27}$ selon ses compétences.

\subsubsection{Conception des stratégies et actions pour le développement}

Cette étape a permis de valoriser les acquis du processus de concertation et de partenariat mis en place entre les chercheurs, les acteurs locaux et les partenaires régionaux durant la période d'élaboration du diagnostic biophysique et socio-économique de la région de Béni Khédache.

Comme cela a été le cas lors de la phase de diagnostic de la région, les mêmes partenaires, mais cette fois-ci avec la contribution des développeurs, des planificateurs et des aménageurs, se sont penchés sur le diagnostic afin d'en tirer des orientations et des idées sur les éventuelles solutions et actions à entamer en faveur de la région et visant l'amélioration des différents aspects qui ont trait à la vie de la population locale. Le résultat a été la production d'un vrai cadre stratégique pour l'initiation d'un processus de développement durable dans cette "zone difficile".

En partant du tableau de problématisation de la région de Béni Khédache et des différentes observations et informations recueillies sur le territoire, les agronomes, économistes et sociologues se sont mis d'accord sur des solutions et orientations à suivre pour chaque objectif et problème spécifique (tableau 2), afin d'améliorer la situation de vie des habitants de la région. Ceci n'a pu être concrétisé qu'avec l'appui des personnes formées et expérimentées dans le domaine du développement rural soit principalement des planificateurs et des aménageurs.

$27 \quad$ Christine Partoune, L'approche interdisciplinaire, 1999, [en ligne] http://www. lmg.ulg.ac.be/competences/chantier/contenus/cont_interdis.html, consulté en février 2011. 


\section{Tableau 2}

Orientations et solutions aux problèmes posés dans la phase du diagnostic de la région

\begin{tabular}{|c|c|c|}
\hline $\begin{array}{l}\text { Renforcement du secteur } \\
\text { économique }\end{array}$ & $\begin{array}{l}\text { Promotion de } \\
\text { l'agriculture }\end{array}$ & $\begin{array}{l}\text { Fixation de la } \\
\text { population et } \\
\text { création des emplois }\end{array}$ \\
\hline $\begin{array}{l}\text { Diversification et pro- } \\
\text { motion des activités non } \\
\text { agricoles génératrices de } \\
\text { revenus : } \\
\text { - Promotion de l'artisa- } \\
\text { nat local } \\
\text { - Promotion du secteur } \\
\text { touristique en faisant } \\
\text { de Béni Khédache une } \\
\text { destination touristique } \\
\text { - Valorisation des res- } \\
\text { sources locales (patri- } \\
\text { moine, paysage, } \\
\text { savoir-faire, etc.) } \\
\text { - Valorisation des res- } \\
\text { sources minières }\end{array}$ & $\begin{array}{l}\text { Renforcement et } \\
\text { consolidation des } \\
\text { travaux de LCD* et } \\
\text { de CES } \\
>\text { Promotion de la pro- } \\
\text { duction agricole avec } \\
\text { un cachet local (pro- } \\
\text { duits du terroir) } \\
\text { Amélioration des } \\
\text { parcours pour renfor- } \\
\text { cer l'activité d'élevage } \\
\text { Amélioration de la } \\
\text { productivité agricole } \\
>\text { Valorisation des sous- } \\
\text { produits agricoles et } \\
\text { d'élevage (bois, cuir, } \\
\text { fromage et beurre, } \\
\text { etc.) }\end{array}$ & $\begin{array}{l}\text { Cet objectif est la } \\
\text { résultante normale de } \\
\text { l'amélioration des } \\
\text { deux secteurs agricole } \\
\text { et économique }\end{array}$ \\
\hline
\end{tabular}

* LCD : Lutte contre la désertification

De même que le premier volet en lien avec le diagnostic et la problématisation de la région d'étude, les solutions et propositions dans ce volet résultent d'un travail de collaboration et de concertation entre les différents acteurs concernés par le développement de Béni Khédache. L'interdisciplinarité étant la méthode à suivre lors de cette démarche de développement rural.

Les résultats obtenus suite à ce travail ont été à l'origine du lancement d'un grand nombre de projets et d'activités inspirés des orientations apportées par le groupe des chercheurs et des institutions ayant travaillé en collaboration pour un meilleur avenir de la région nécessiteuse de Béni Khédache. C'est ainsi que depuis quelques années, la région connait une dynamique socio-économique sans précédent marquée par le développement du secteur du tourisme rural et de l'artisanat bédouin; ces 
deux secteurs viennent renforcer une activité agricole de plus en plus orientée vers la production du terroir ${ }^{28}$.

C'est donc grâce à une démarche du développement rural basée sur une approche interdisciplinaire au niveau d'une zone défavorisée, que la situation commence à évoluer vers le meilleur. Le développement rural ne peut être imaginé, en fait, que selon une vision générale prenant en considération les différents problèmes présents sur le territoire et qui constituent un obstacle à sa prospérité. Par ailleurs, c'est en raison de la complexité de ces problèmes qu'il faut chercher les solutions à apporter auprès de différentes disciplines traitant chacune d'un problème précis.

\section{Conclusion}

L'interdisciplinarité comme méthode de recherche s'impose dès le moment où on traite de sujets ou de problématiques complexes qui nécessitent une vision globale. C'est, en fait, à partir du recours à différentes disciplines, aux objets d'étude, aux théories et à des méthodologies variés qu’on évite la ségrégation des éléments composant l'information et que l'on en arrive à des réflexions et idées plus productives.

La discipline, de par sa spécialisation dans un seul savoir, se trouve souvent incapable de traiter un sujet ou d'étudier un phénomène sans recourir à d'autres disciplines. C'est ainsi qu'elle franchit ses limites pour chercher l'information dans d'autres savoirs. L'intégration des analyses et travaux émanant des différentes disciplines impliquées est garante de la résolution des problèmes qui les chevauchent.

Cet article traite de la notion d'interdisciplinarité; il définit cette dernière et justifie son utilité dans les différents champs de travail scientifique. Il rappelle, par ailleurs, quelques aspects théoriques liés à cette méthode de recherche. Ainsi apparaît l'interdisciplinarité comme ingénierie systémique où il faut se

28 Pour plus de détails voir la thèse de Sana Moussalim, Contribution à l'évaluation des projets de développement rural local: le projet "Jessour et Ksour de Béni Khédache ", Master of science, Institut agronomique méditerranéen de Montpellier, 2009, [en ligne] http://ressources.iamm.fr/theses/96.pdf, consulté en décembre 2010. 
rapporter à tous les éléments du système dans lequel s'insère l'objet à l'étude.

On avait rapporté l'exemple du développement rural comme approche interdisciplinaire. Ce processus, défini dans le texte comme savoir interdisciplinaire, appelle à prendre en considération toutes les dimensions du territoire à développer et à les intégrer dans une démarche globale visant l'amélioration des aspects socio-économiques de la zone en question. Cette vision implique nécessairement la contribution de disciplines diverses dans la conception de schémas de développement et le déroulement des actions qui en résultent.

\section{Bibliographie}

Abichou, Hanen, Mongi Sghaier et Anne-marie Jouve, "Quelle alternative pour le développement des zones défavorisées en Tunisie ", New Medit, vol. $7, \mathrm{n}^{\circ} 1$, mars 2008 , p. 38-44.

Bertalanffy, Ludwig Von, Théorie générale des systèmes, Paris, Dunod/ Systémique, 1993, 308 p.

Carte de la Tunisie (2010), [en ligne] http://www.routard.com/guide_carte/ code_dest/tunisie.htm, consulté le 13 décembre 2010.

Crozier, Michel et Erhard Friedberg, L'acteur et le système, les contraintes de l'action collective, Paris, Seuil, 1992, 500 p.

De Rosnay, Joël, Le macroscope (Vers une vision globale), Paris, Seuil, 1975, $295 \mathrm{p}$.

Descartes, René, Discours de la méthode, Paris, Hachette, 1856, 53 p., [en ligne] http://books.google.fr, consulté le 15 janvier 2011.

Donnadieu, Gérard, Daniel Durand, Danièle Neel, Emmanuel Nunez et Lionel Saint-Paul, Lapproche systémique : de quoi s’agit-il, [en ligne] http://www.afscet.asso.fr/SystemicApproach.pdf, consulté en décembre 2010 [2003].

Durand, Daniel, La systémique, Paris, PUF, [1979] 1998, 121 p.

Elloumi, Mohamed, « Politiques du développement rural en Tunisie, acquis et perspectives ", Options Méditerranéennes, Sér. A / n 71, 2006, p. 55-65.

ICRA, (Centre international pour la recherche agricole) équipe interdisciplinarité, Interdisciplinarité, concepts-clés, 2008, [en ligne] http://www. 
icra-edu.org/objects/francolearn/ACFA0.pdf, consulté le 3 décembre 2010.

Institut des Régions Arides (IRA) et Coopération Technique Allemande (GTZ), Plan d'action local de lutte contre la désertification (PALLCD) dans la délégation de Béni Khédache (Gouvernorat de Médenine), Ministère de l'environnement et du développement durable, 2007, $135 \mathrm{p}$.

Lugan, Jean Claude, La systémique sociale, Paris, PUF, 2005, 127 p.

Morin, Edgar, Sur l'interdisciplinarité, [en ligne] http://www.mcxapc.org/ docs/conseilscient/morin_interdisciplinarite_021103.pdf, consulté le 15 novembre 2010 [2003].

Morin Edgar et Jean-Louis Le Moigne, L’intelligence de la complexité, Paris, Harmattan, 1999, 332 p.

Moussalim, Sana, Contribution à l'évaluation des projets de développement rural local : le projet "Jessour et Ksour de Béni Khédache ", Master of sciences, Institut agronomique méditerranéen de Montpellier, 2009, $191 \mathrm{p}$.

Nissani, Moti, «Fruits, Salads, and Smoothies: A Working Definition of Interdisciplinarity ", Journal of Educational Thought, vol. 29, 1995, p. 119-126.

Partoune, Christine, L'approche interdisciplinaire, [en ligne] http://www. lmg.ulg.ac.be/competences/chantier/contenus/cont_interdis.html, consulté en février 2011 [1999].

Sghaier, Mongi, Ali Abaab et Pierre Campagne, "L'expérimentation de nouvelles approches de développement local et de gestion participative des ressources naturelles ", dans Didier Genin, Henri Guillaume, Mohammed Ouessar, Azalez Ouled Belgacem, Bruno Romagny, Mongi Sghaier et Houcline Taamallah (dir.), Entre désertification et développement. La Jeffara tunisienne, Tunis, Éditions Cérès, IRD et IRA, 2006, p. 303-313.

Sghaier, Mongi et Ali Abbab, "L'inscription de la thématique du colloque DELZOD dans le parcours scientifique du laboratoire d'Économie et sociétés rurales ", Cadrage du colloque international "Sociétés en transition et développement local en Zones difficiles, DELZOD " Médenine, IRA, 2009.

Simony, Nadia, Relais pour l'emploi : solidaires et acteurs de leur reclassement, Paris, Harmattan, 1998, 140 p.

Wikipédia, L'encyclopédie libre en ligne (2011), Approche systémique, [en ligne] http://fr.wikipedia.org/wiki/Approche_systémique, consulté en janvier 2011. 